Bio - grafía. Escritos sobre la Biología y su Enseñanza. ISSN 2027-1034

Edición Extraordinaria. p.p. 330 - 340

Memorias del IX Encuentro Nacional de Experiencias en Enseñanza de la Biología y la

Educación Ambiental. IV Congreso Nacional de Investigación en Enseñanza de la Biología.

\title{
LA PREGUNT A COMO ESTRATEGIA DIDÁCTICA PARA EL APRENDIZAJE SIGNIFICATIVO DEL CONCEPTO HERENCIA BIOLÓGICA, EN ESTUDIANTES DE GRADO OCTAVO
}

\section{THE QUESTION AS A DIDACTIC STRATEGY FOR MEANINGFUL LEARNING OF THE BIOLOGICAL INHERIT ANCE CONCEPT, IN EIGHTH GRADE STUDENTS}

\author{
Conde, Juan'; Bernal, Johanna ${ }^{2}$
}

\section{RESUMEN:}

En la enseñanza de la genética el concepto de herencia biológica resulta ser uno de los temas más difíciles de enseñar y aprender en el aula de clase, esto puede deberse a la naturaleza de los conceptos de esta disciplina, las estrategias didácticas implementadas y por último a los conocimientos y formas de razonamiento de los alumnos. En el presente trabajo se presenta los resultados obtenidos de una unidad didáctica denominada "¿Cómo se expresan mis genes?", fundamentada en los principios del aprendizaje significativo e implementada en la Institución Educativa Distrital Colegio Chuniza en estudiantes de grado octavo, para ello se desarrollaron estrategias de carácter interpretativo, haciendo uso de herramientas contempladas en la investigación cualitativa (grabaciones de audio, transcripciones de clase, observaciones y diario del profesor). La investigación educativa tuvo como fin reconocer de qué manera la pregunta docente-estudiante puede constituirse en una estrategia de aprendizaje significativo para la comprensión de conceptos de herencia biológica, de tal manera que permita construir un mayor y mejor aprendizaje acerca de los procesos relacionados con la transmisión de la información hereditaria. Los resultados obtenidos mostraron que las preguntas formuladas por los docentes en formación, contribuyen de manera sistemática a la apropiación conceptual en los estudiantes, a su vez, estas preguntas favorecieron en los estudiantes la habilidad para formular preguntas y la modificación de las ideas previas.

PALABRAS CLAVE: Herencia biológica, aprendizaje significativo, carácter interpretativo, diario del profesor, observaciones, preguntas.

\footnotetext{
${ }^{1}$ Estudiante, Licenciatura en biología, Universidad Distrital Francisco José de Caldas, Facultad de ciencias y educación, correo: jgcondep@correo.udistrital.edu.co

2 Estudiante, Licenciatura en biología, Universidad Distrital Francisco José de Caldas, Facultad de ciencias y educación, correo: ijbernalp@correo.udistrital.edu.co
} 
Bio - grafía. Escritos sobre la Biología y su Enseñanza. ISSN 2027-1034

Edición Extraordinaria. p.p. 330 - 340

Memorias del IX Encuentro Nacional de Experiencias en Enseñanza de la Biología y la

Educación Ambiental. IV Congreso Nacional de Investigación en Enseñanza de la Biología.

\begin{abstract}
In the teaching of genetics the concept of biological inheritance turns out to be one of the most difficult subjects to teach and learn in the classroom, this can be due to the nature of the concepts of this discipline, the didactic strategies implemented and finally to the knowledge and forms of reasoning of the students. This paper presents the results obtained from a didactic unit called "¿Cómo se expresan mis genes?", based on the principles of meaningful learning and implemented in the educational institution district Colegio Chuniza in Eighth grade students, for this purpose, developed strategies of an interpretative nature, using tools contemplated in qualitative research (audio recordings, transcripts of class, observations and Journal of the teacher). The educational research was aimed at recognizing how the teaching-student question can be a meaningful learning strategy for understanding biological inheritance concepts, in such a way that it allows to build a greater and better learning about the processes related to the transmission of hereditary information. The results showed that the questions asked by the teachers in training, systematically contribute to the conceptual appropriation in the students, in turn, these questions favored in the students the ability to formulate questions and the modification of the previous ideas.
\end{abstract}

KEY WORDS: Biological inheritance, meaningful learning, interpretative character, teacher's diary, observations, questions.

\title{
INTRODUCCIÓN
}

El presente artículo de investigación educativa se desarrolló bajo el enfoque pedagógico de aprendizaje significativo, planteado por Ausubel (1978), en el cual menciona que un nuevo conocimiento o información se relaciona con la estructura cognitiva del que aprende de forma no arbitraria y sustantiva o no literal. Esa interacción con la estructura cognitiva no se produce considerándola como un todo, sino con aspectos relevantes presentes en la misma, que reciben el nombre de subsumidores o ideas de anclaje (Ausubel, 1976). La presencia de ideas, conceptos o proposiciones inclusivas, claras y disponibles en la mente del estudiante es lo que dota de significado a ese nuevo contenido en interacción con el mismo. Pero no se trata de una simple unión, sino que en este proceso los nuevos contenidos adquieren significado para el sujeto produciéndose una transformación de los subsumidores de su estructura cognitiva, que resultan así progresivamente más diferenciados, elaborados y estables (Ausubel, 1976).

Teniendo en cuenta que mediante la cognición se procesan representaciones de la realidad, las preguntas pueden considerarse el vehículo a través de cual se activan y se orientan las estrategias cognitivas como la comprensión, la representación, la aplicación y la investigación (Montenegro, 2001). Por consiguiente, generar buenas preguntas permite 
Bio - grafía. Escritos sobre la Biología y su Enseñanza. ISSN 2027-1034

Edición Extraordinaria. p.p. 330 - 340

Memorias del IX Encuentro Nacional de Experiencias en Enseñanza de la Biología y la

Educación Ambiental. IV Congreso Nacional de Investigación en Enseñanza de la Biología.

que el estudiante desarrolle un aprendizaje activo, promoviendo la construcción de su propio conocimiento y metacognición (Márquez y Roca, 2006)

En el proceso de aprendizaje, la formulación de preguntas tienen un papel primordial, tanto las preguntas que plantea el profesorado como las que se plantea el propio alumno. Pero no todas las preguntas contribuyen del mismo modo al aprendizaje. Las preguntas utilizadas en el aula, como toda actividad de enseñanza-aprendizaje pueden tener distintos objetivos. Ello implica la necesaria coherencia entre la forma de la pregunta y el objetivo que se pretende (Márquez y Roca, 2004). Las preguntas elaboradas por los estudiantes, deben ser para el profesor un indicador que este estudiante ha pensado en las ideas discutidas, y que está intentando encontrar una forma de vincularlas a otros conocimientos que posee, demostrando de cierta forma una brecha existente entre lo que él sabe y hacia donde intenta llegar. En una investigación realizada Chin y Osborne (2008), mencionan que la formulación de preguntas, es una habilidad metacomprensiva, como lo es la argumentación, que necesita ser desarrollada, pues no se genera de forma espontánea. La competencia de elaborar preguntas está compuesta de procesos cognitivos y lingüísticos complejos que deberían ser tenidos en cuenta, al momento de diseñar actividades de instrucción especifica (Montenegro, 2001).

La complejidad del estudio de la genética en la enseñanza secundaria deriva, en buena medida, de la naturaleza de sus conceptos, pero se ve notablemente incrementada por la necesidad de aplicarlos a estrategias de aprendizaje, complejas en sí mismas, como la formulación de preguntas y la resolución de problemas. Esta clase de actividades puede servir para comprender mejor la estructura conceptual de la genética y la naturaleza de la ciencia como actividad intelectual y para desarrollar algunas destrezas propias de esta disciplina, como el ensayo de determinadas hipótesis y otras de carácter más general, como redescribir datos de un problema, búsqueda de información, análisis de datos y resultados (Benítez, 2013)

Se han realizado algunas investigaciones didácticas, en las cuales se ha puesto de manifiesto que los estudiantes tienen dificultades para entender muchos conceptos sobre genética, así como acerca de los mecanismos relacionados con la transmisión de la herencia biológica (Quijano et. al., 2005). Se hace necesario reflexionar sobre el origen de los obstáculos que los estudiantes encuentran en el aprendizaje de esta materia, así como proponer metodologías innovadoras en la enseñanza de la genética (Caballero, 2008). La enseñanza de la información hereditaria se inicia, en nuestro sistema educativo, en los niveles de enseñanza secundaria. Se supone que, hasta este momento los estudiantes no han desarrollado los esquemas de razonamiento necesarios, ni poseen los conocimientos pertinentes que le permitan comprender los conceptos elementales de genética. Aun reconociendo las dificultades que éstos plantean (a los profesores para enseñarlos, y a los alumnos para aprenderlos), existe una amplia coincidencia entre el profesorado de biología en la necesidad de incluir contenidos básicos sobre la herencia en estos niveles educativos (Banet y Ayuso, 1995). Sus resultados destacan la importancia que los profesores de enseñanza secundaria conceden a la genética mendeliana, a la teoría cromosómica de la herencia o al concepto de gen, entre otros. Sin 
Bio - grafía. Escritos sobre la Biología y su Enseñanza. ISSN 2027-1034

Edición Extraordinaria. p.p. 330 - 340

Memorias del IX Encuentro Nacional de Experiencias en Enseñanza de la Biología y la

Educación Ambiental. IV Congreso Nacional de Investigación en Enseñanza de la Biología.

embargo, como en otras áreas de la biología, muchos trabajos de investigación han puesto de manifiesto que, después de la instrucción, los aprendizajes de los estudiantes sobre la herencia biológica son menos significativos de lo que cabría esperar (Ayuso y Banet, 2002).

\section{METODOLOGÍA}

El presente trabajo se realizó bajo los fundamentos de la investigación cualitativa, que como menciona Creswell (1998) se basa en un proceso de indagación interpretativa, apoyado en técnicas metodológicas tales como la teoría fundamentada en datos y recolección de una variedad de materiales empíricos.

Se realizó una unidad didáctica acudiendo a los conceptos establecidos por Fonseca (2012), esta unidad se tituló “¿Cómo se expresan mis genes?", se implementó en la Institución Educativa Distrital Colegio Chuniza en la localidad de Usme, Bogotá D,C. con estudiantes de estratos 1 y 2 . El grupo de estudiantes con quienes se llevó a cabo la investigación corresponde al grado 802 de la jornada tarde, conformado por 36 alumnos (17 niñas y 19 niños) con edades entre 12 y 15 años. El recurso principal de la investigación fue la formulación de preguntas docentes-estudiantes, con respecto al concepto de herencia biológica.

En la etapa de implementación, la cual constó de cinco sesiones, se reconocieron e identificaron las ideas previas que tenían los estudiantes con respecto a la herencia biológica, por medio de preguntas que abordaron temas desde ciclo celular, hasta variabilidad genética. En el transcurso de las sesiones, se realizaron diferentes actividades, las cuales permitieron que los estudiantes se generaran preguntas, a partir de las intervenciones didácticas de los docentes en formación. Para la obtención de la información del trabajo, se tuvo en cuenta el uso de herramientas contempladas en la investigación cualitativa tales como grabaciones de audio, transcripciones de clase, observaciones y diario del profesor.

\section{RESULTADOS}

\begin{tabular}{|l|l|l|}
\hline Cód. & $\begin{array}{l}\text { Objetivos de } \\
\text { pregunta }\end{array}$ & Preguntas formuladas por los docentes en formación \\
\hline PRI & Encontrar respuesta & ¿Qué entienden por información hereditaria? \\
& & ¿Qué conocen o qué comprenden ustedes sobre el ADN? \\
& & ¿Qué creen ustedes que es el ciclo celular? \\
& ¿Por qué creen que la información hereditaria está en los \\
\hline
\end{tabular}


Bio - grafía. Escritos sobre la Biología y su Enseñanza. ISSN 2027-1034

Edición Extraordinaria. p.p. 330 - 340

Memorias del IX Encuentro Nacional de Experiencias en Enseñanza de la Biología y la

Educación Ambiental. IV Congreso Nacional de Investigación en Enseñanza de la Biología.

\begin{tabular}{|l|l|l|}
\hline \multirow{1}{*}{} & gametos? \\
& & ¿Qué sería para ustedes un carácter? \\
& ¿Qué células creen que llevan genes? \\
& ¿Qué es un gen? \\
& ¿En dónde se originan los cromosomas? \\
& ¿Qué es un cruce genético?
\end{tabular}


Bio - grafía. Escritos sobre la Biología y su Enseñanza. ISSN 2027-1034

Edición Extraordinaria. p.p. 330 - 340

Memorias del IX Encuentro Nacional de Experiencias en Enseñanza de la Biología y la

Educación Ambiental. IV Congreso Nacional de Investigación en Enseñanza de la Biología.

Tabla 1. Clasificación y objetivos de las preguntas formuladas por los docentes en formación (Chin y Chia, 2004).

Hacer uso de la pregunta para el aprendizaje significativo de la herencia biológica, generó un mayor interés en los estudiantes, ya que constituyó una de las propuestas más coherentes con la construcción del conocimiento científico de este campo. El anális is de la presente investigación, se hizo a partir del discurso grupal que realizaron los docentes en formación y los estudiantes, reconociendo así, los diferentes tipos de preguntas formulados en el aula. Se pudo evidenciar, que en las cinco sesiones de clases, la mayoría de las preguntas elaboradas por los docentes en formación fueron de tipo abiertas (ver Tabla 1), este modelo de pregunta en el ámbito del aprendizaje significativo motiva a los estudiantes a exponer de manera indefinida los conocimientos previos que poseen sobre la genética y los mecanismos relacionados con la herencia biológica. En un estudio realizado por Bermúdez \& De Longhi (2011), el hacer uso de la pregunta para el aprendizaje significativo, ayuda a los alumnos a aumentar el nivel de comprensión y a abordar los contenidos en niveles de complejidad creciente. Asimismo, los resultados obtenidos de las investigaciones realizadas por Chin y Chia (2004), acreditan que el uso de preguntas de alto nivel cognitivo, realizado por el docente en el aula, tienen un efecto positivo sobre el aprendizaje significativo del estudiantado.

Las preguntas que se obtuvieron a partir de la intervenciones didácticas, se clasificaron de acuerdo al sistema de clasificación de preguntas propuesto por Chin y Chia (2004) (Ver tabla 1). Estas preguntas que se formularon en el aula de clase, a su vez se categorizaron en cuatro tipos de preguntas: Preguntas de recopilación de información (PRI), que son preguntas que buscan una información objetiva básica; preguntas puente (PP), que son preguntas que intentan encontrar conexiones entre conceptos; preguntas de extensión (PE), las cuales son preguntas de exploración más allá del alcance de los problemas propuestos, y a su vez son resultantes de la creatividad o de la aplicación de conocimientos recién adquiridos; y por último, preguntas evaluativas y criticas (PEC), las cuales contribuyen a la toma de decisiones o cambio cognitivo (Chin y Chia, 2004). Con el uso de estas preguntas, se logró que los estudiantes se acercaran al conocimiento de la genética y los mecanismos de herencia biológica, y que además, adquirieran una actitud crítica y reflexiva frente al mismo. De esta forma, el hacer uso de la pregunta, como una estrategia didáctica para el aprendizaje significativo, se convierte en una práctica dialógica, constructiva y metacognitiva que va introduciendo al estudiante en los procesos de investigación (Montenegro, 2001).

A partir de las intervenciones didácticas de los docentes en formación, los estudiantes formularon gran cantidad de preguntas, que permitieron dar cuenta de manera sistemática la evolución en las concepciones desarrolladas en el aula. A continuación se mostrarán dichas preguntas de acuerdo con el orden de las sesiones realizadas:

\begin{tabular}{|c|l|l|}
\hline Sesión & Preguntas formuladas por los estudiantes & Fases de aprendizaje significativo \\
\hline $\mathbf{1}$ & $\begin{array}{l}\text { Estudiante 1: “¿Por qué mi hermana tiene } \\
\text { los ojos del color de mi mama y de mi papá, }\end{array}$ & Fase inicial de aprendizaje: \\
\hline
\end{tabular}


Bio - grafía. Escritos sobre la Biología y su Enseñanza. ISSN 2027-1034

Edición Extraordinaria. p.p. 330 - 340

Memorias del IX Encuentro Nacional de Experiencias en Enseñanza de la Biología y la

Educación Ambiental. IV Congreso Nacional de Investigación en Enseñanza de la Biología.

\begin{tabular}{|c|c|c|}
\hline & $\begin{array}{l}\text { se mezclan?” } \\
\text { Estudiante 2: “QQué pasa cuando la mamá } \\
\text { tiene una enfermedad y el papá no?” } \\
\text { Estudiante 3: “ ¿Por qué mis hermanos son } \\
\text { más altos que yo?” } \\
\text { Estudiante 4: “¿qué pasa cuando una } \\
\text { persona de piel morena se cruza con una } \\
\text { persona de piel blanca?” } \\
\text { Estudiante 5: “por qué algunas veces nos } \\
\text { parecemos más al papá o a la mamá?” } \\
\text { Estudiante 6: “ilas flores rosadas son } \\
\text { producto de una flor roja y blanca?” }\end{array}$ & $\begin{array}{l}\text { 1. En esta fase, los estudiantes gradualmente } \\
\text { fueron construyendo un panorama global acerca } \\
\text { de los conceptos básicos sobre genética y } \\
\text { mecanismos de herencia biológica. Para lo cual, } \\
\text { usaron sus conocimientos esquemáticos, } \\
\text { estableciendo analogías con otros dominios que } \\
\text { ya conocen mejor. Para la representación de ese } \\
\text { nuevo dominio, construyeron suposiciones } \\
\text { basadas en experiencias previas, etc. }\end{array}$ \\
\hline 2 & $\begin{array}{l}\text { Estudiante 1: “¿Los alelos, son una } \\
\text { estructura aparte, o son el mismo gen?" } \\
\text { Estudiante 2: “¿los cromosomas no son } \\
\text { como una célula?” } \\
\text { Estudiante 3: “¿Eso quiere decir que los } \\
\text { cromosomas se forman a partir del ciclo } \\
\text { celular?” } \\
\text { Estudiante 1: “¿mitosis y meiosis no es lo } \\
\text { mismo?” } \\
\text { Estudiante 2: “ ¿gracias al ciclo celular } \\
\text { meiótico es que todos los seres vivos somos } \\
\text { tan diferentes?" } \\
\text { Estudiante 3: “Los genes de las células } \\
\text { germinales son las únicas que se pueden } \\
\text { heredar?” } \\
\text { Estudiante 4: “Qué pasa con los genes de } \\
\text { las células somáticas?” } \\
\text { Estudiante 5: “QQuién determina el sexo, el } \\
\text { papá o la mamá?” }\end{array}$ & $\begin{array}{l}\text { Fase inicial de aprendizaje: } \\
\text { 2. Continuando con esta primera fase, la } \\
\text { información de las preguntas que formularon los } \\
\text { estudiantes es global. La información aprendida } \\
\text { es concreta y vinculada al contexto específico. Se } \\
\text { evidencia además que en esta sesión, los } \\
\text { estudiantes hacen un uso predominante de } \\
\text { estrategias de repaso para aprender la } \\
\text { información. Sin embargo, hubo un escaso } \\
\text { conocimiento sobre los conceptos básicos de } \\
\text { ciclo celular. }\end{array}$ \\
\hline
\end{tabular}


Bio - grafía. Escritos sobre la Biología y su Enseñanza. ISSN 2027-1034

Edición Extraordinaria. p.p. 330 - 340

Memorias del IX Encuentro Nacional de Experiencias en Enseñanza de la Biología y la

Educación Ambiental. IV Congreso Nacional de Investigación en Enseñanza de la Biología.

\begin{tabular}{|c|c|c|}
\hline 3 & $\begin{array}{l}\text { Estudiante 1: “¿El ADN no se encuentra } \\
\text { solo en la sangre?” } \\
\text { Estudiante 2: “¿Entonces cualquier célula } \\
\text { de mi cuerpo siempre va a tener ADN?” } \\
\text { Estudiante 3: “¿Por qué encontramos ADN } \\
\text { en la saliva si eso no es una célula?” } \\
\text { Estudiante 4: “¿Por qué se hace extracción } \\
\text { de ADN vegetal con frutas?” } \\
\text { Estudiante 5: ¿Cómo los genes actúan en la } \\
\text { fruta? }\end{array}$ & $\begin{array}{l}\text { 3. Ya en la fase intermedia, los estudiantes por } \\
\text { medio de sus preguntas empiezan a encontrar } \\
\text { relaciones y similitudes entre las partes aisladas y } \\
\text { llegan a configurar esquemas y mapas cognitivos } \\
\text { acerca de todo lo relacionado con ciclo celular y } \\
\text { ADN, mejorando su aprendizaje en forma } \\
\text { progresiva. Sin embargo, estos esquemas no } \\
\text { permiten aún que los estudiantes se conduzcan } \\
\text { en forma automática o autónoma. Se va } \\
\text { realizando de manera paulatina un procedimiento } \\
\text { más profundo del concepto. El conocimiento } \\
\text { aprendido se vuelve aplicable a otros contextos. }\end{array}$ \\
\hline 4 & 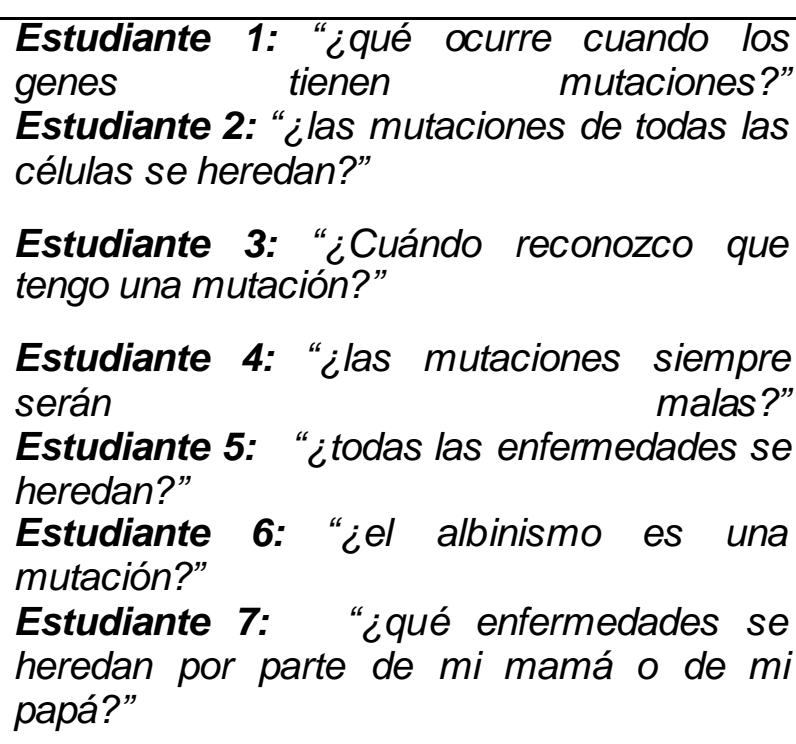 & $\begin{array}{l}\text { Fase intermedia de aprendizaje } \\
\text { 4. En esta fase, se puede evidenciar que hay más } \\
\text { oportunidad para reflexionar sobre los } \\
\text { mecanismos relacionados con la herencia } \\
\text { biológica. El conocimiento llega a ser más } \\
\text { abstracto, es decir, menos dependiente del } \\
\text { contexto donde originalmente fue adquirido. En } \\
\text { este punto, los estudiantes, empiezan a asociar } \\
\text { las enfermedades con procesos que se } \\
\text { desarrollaron en sesiones anteriores, lo que } \\
\text { quiere decir que empieza a haber una articulación } \\
\text { del tema. }\end{array}$ \\
\hline 5 & $\begin{array}{l}\text { Estudiante 1: "Si en el cruce de una persona } \\
\text { de piel negra con una de piel blanca, el bebé } \\
\text { sale más del color negro, ¿quiere decir que } \\
\text { el gen dominante es el de la persona de piel } \\
\text { negra, y recesivo el de la persona de piel } \\
\text { blanca?" } \\
\text { Estudiante 2: "¿Qué hace que seamos tan } \\
\text { diferentes a cos demás?" } \\
\text { Estudiante 3: "¿las crías de mis perros } \\
\text { tienen manchas blancas y manchas negras, }\end{array}$ & $\begin{array}{l}\text { Fase terminal de aprendizaje: } \\
\text { 5. Por último, los conocimientos que comenzaron } \\
\text { a ser elaborados en esquemas o mapas } \\
\text { cognitivos en la fase inicial, llegan a estar más } \\
\text { integrados y a funcionar con mayor autonomía. } \\
\text { Las preguntas planteadas en esta fase, articulan } \\
\text { todos los conceptos vistos a lo largo de las } \\
\text { sesiones anteriores, produciéndose una } \\
\text { transformación de los subsumidores de su }\end{array}$ \\
\hline
\end{tabular}


Bio - grafía. Escritos sobre la Biología y su Enseñanza. ISSN 2027-1034

Edición Extraordinaria. p.p. 330 - 340

Memorias del IX Encuentro Nacional de Experiencias en Enseñanza de la Biología y la

Educación Ambiental. IV Congreso Nacional de Investigación en Enseñanza de la Biología.

\begin{tabular}{|l|l|l|}
\hline $\begin{array}{l}\text { eso podría considerarse codominancia?” } \\
\text { Estudiante 4: “yo me parezco a mi mamá } \\
\text { y a mi papá por igual, eso sería herencia } \\
\text { intermedia?” }\end{array}$ & $\begin{array}{l}\text { estructura cognitiva (Ausubel, 1976). Existe } \\
\text { mayor énfasis en esta fase sobre la ejecución del } \\
\text { aprendizaje, dado que los cambios en la } \\
\text { ejecución que ocurren se deben a variaciones } \\
\text { provocadas por las intervenciones didácticas del } \\
\text { docente. }\end{array}$ \\
\hline
\end{tabular}

Tabla 2. Preguntas formuladas por los estudiantes a partir de las intervenciones didácticas de los docentes en formación.

Partiendo de las posturas conceptuales sobre la definición de las estrategias para el aprendizaje significativo, se plantea que a través de las preguntas formuladas por los estudiantes, hubo una aproximación a los enfoques teóricos de Ausubel (1976); quien menciona que las teorías para el aprendizaje son la gama de conocimientos organizados sistemáticamente, cuya finalidad es proporcionar una visión de conjunto para el proceso de aprendizaje, que involucra pasar de meras afirmaciones, al desarrollo de la predicción, la experimentación y la explicación. Las preguntas formuladas por los alumnos, se realizaron como producto de la "mutua dependencia" entre los procesos cognitivos individuales de reconstrucción de los saberes disciplinares y las variables propias del contexto didáctico (Castorina, 2010). Las modificaciones cognoscitivas "no ocurren por fuera de las situaciones didácticas, sino en el encuentro de los saberes previos y los saberes disciplinares entre los que media el docente. Las preguntas formuladas por los docentes en formación también favorecieron a la generación de una cascada de actividades cognitivas, que les permitieron a los estudiantes ir construyendo a través de piezas su conocimiento, y resolver conflictos de comprensión (Chin y Osborne, 2008).

\section{CONCLUSIONES}

-Esta experiencia educativa en el aula, evidencia que las preguntas formuladas por los docentes en formación, contribuyeron a que los estudiantes expusieran sus conocimientos previos, a partir de las intervenciones didácticas. Por lo tanto, la pregunta docenteestudiante, en la enseñanza de la herencia biológica, abordó las perspectivas principales que se emplean en el aprendizaje significativo.

-En función del análisis de los resultados, la fundamentación teórica y los objetivos propuestos, se observó que el uso de la pregunta como estrategia para el aprendizaje significativo en la enseñanza de la herencia biológica, fomentó a la discusión y al debate, aumentando la calidad del discurso y la participación en el aula. A su vez, se pudo evidenciar en las sesiones desarrolladas, que los estudiantes a través de sus preguntas relacionaban la información nueva con la que ya poseían, es decir con la estructura cognitiva ya existente. 
Bio - grafía. Escritos sobre la Biología y su Enseñanza. ISSN 2027-1034

Edición Extraordinaria. p.p. 330 - 340

Memorias del IX Encuentro Nacional de Experiencias en Enseñanza de la Biología y la

Educación Ambiental. IV Congreso Nacional de Investigación en Enseñanza de la Biología.

-Lo estudiantes de grado octavo de la Institución Educativa Distrital Colegio Chuniza, inicialmente poseían un escaso conocimiento con respecto a conceptos básicos, como cromosomas, genes, alelos o mutaciones y no alcanzaban a comprender el significado de procesos importantes, como la meiosis, o los mecanismos que se llevan a cabo en la transmisión de los caracteres hereditarios. Pero estas concepciones pasaron de ser meras afirmaciones erróneas a un verdadero aprendizaje significativo, gracias al uso de preguntas que promovieron a la búsqueda de información, investigación, formulación de hipótesis y problematización.

\section{REFERENCIAS}

-Ausubel, D. P. (1976). Psicología educativa. Un punto de vista cognoscitivo. Ed. Trillas. México 190-199.

-Ausubel, D.P., Novak, J.D. \& Hanesian, H. (1978). Educational psychology: a cognitive view. New York: Holt, Rinehart and Winston. Publicado en portugués por la Editora Interamericana, Rio de Janeiro, 1980, 211-239.

-Ayuso, G. E., Banet, E. (2002) Alternativa a la enseñanza de la genética en educación secundaria. Departamento de Didáctica de las Ciencias Experimentales, Enseñanza de las ciencias, 133-154.

-Bermúdez, G. M. A. \& De Longhi, A. L. (2011). Niveles de comprensión del equilibrio químico en estudiantes universitarios a partir de diferentes estrategias didácticas. Revista Electrónica de Enseñanza de las Ciencias, 10(2), 264-88.

-Banet, E., Ayuso, E. (1995) Introducción a la genética en la enseñanza secundaria y bachillerato: contenidos de enseñanza y conocimientos de los alumnos. Facultad de Educación, Departamento de Didáctica de las Ciencias Experimentales, 137-152.

-Benítez, R. A., (2013) La enseñanza de la genética en el grado noveno de básica secundaria: una propuesta didáctica a la luz del constructivismo. Universidad Nacional de Colombia- sede Medellín, facultad de ciencias, 28-30.

-Caballero, M. (2008). Algunas Ideas Del Alumno De Secundaria Sobre Conceptos Básicos De Genética. Enseñanza De Las Ciencias, 26(2), 227-244.

-Castorina, J. A. (2010) "La epistemología constructivista ante el desafío de los saberes disciplinares". En CASTORINA, J.A. (comp.) Desarrollo del conocimiento social. Prácticas, discursos y teorías. Miño y Dávila, Buenos Aires.

-Chin, C., Chia, L.-G. (2004). Problem-based learning: Using students' questions to drive knowledge construction. Science Education, 88(5), doi: 10.1002/sce.10144, 707-727.

-Chin, C., Osborne, J. (2008). Students' questions: a potential resource for teaching and learning science. Studies in Science Education, 44(1), 1-39 
Bio - grafía. Escritos sobre la Biología y su Enseñanza. ISSN 2027-1034

Edición Extraordinaria. p.p. 330 - 340

Memorias del IX Encuentro Nacional de Experiencias en Enseñanza de la Biología y la Educación Ambiental. IV Congreso Nacional de Investigación en Enseñanza de la Biología.

-Fonseca, G. (2012) La construcción del conocimiento didáctico del contenido en profesores de biología en formación a través del diseño e implementación de unidades didácticas. III Congreso Internacional y VII Nacional de Investigación en educación, pedagogía y formación docente (Capítulo de Memoria) Pp. 1854-1869

-Márquez, C., Roca, M. T. (2004) Plantear preguntas: un punto de partida para aprender ciencias. Revista Educación y Pedagogía, 6-11.

-Márquez, C., Roca, M. T. (2006) La construcción de modelos explicativos complejos mediante preguntas mediadoras. Investigación en la Escuela, 71-81.

-Montenegro, I. A. (2001) Preguntas cognitivas y metacognitivas en el proceso de aprendizaje: Influencia de preguntas cognitivas y metacognitivas en comprensión conceptual y en habilidad para resolver problemas en ciencias, Universidad Pedagógica Nacional. Red Académica, 2-11.

-Quijano, R.; Muela, F. J.; Abril, A.M. (2000) Herencia y genética: concepciones y conocimientos de los estudiantes ( $1^{\text {a }}$ fase), $5-10$. 